\title{
Characterization of subglacial landscapes by a two-parameter roughness index
}

\author{
Xin $\mathrm{LI}^{1,2}$ Bo SUN, ${ }^{1}$ Martin J. SIEGERT, ${ }^{3}$ Robert G. BINGHAM, ${ }^{4}$ Xueyuan TANG, \\ Dong ZHANG, ${ }^{1}$ Xiangbin CUI, ${ }^{1}$ Xiangpei $\mathrm{ZHANG}^{1}$ \\ ${ }^{1}$ Polar Research Institute of China, 451 Jinqiao Road, Pudong, Shanghai 200136, China \\ E-mail: lixin@pric.gov.cn \\ ${ }^{2}$ School of Ocean and Earth Sciences, Tongji University, Shanghai 200092, China \\ ${ }^{3}$ School of GeoSciences, University of Edinburgh, King's Buildings, Edinburgh EH9 3JW, UK \\ ${ }^{4}$ School of Geosciences, University of Aberdeen, Elphinstone Road, Aberdeen AB24 3UF, UK
}

\begin{abstract}
Previous studies using Fourier transformation (FT) methods to analyze subglacial roughness have shown promise for distinguishing between different types of subglacial landscape from raw subglacial elevation data. We derive a two-parameter FT roughness index $\{\xi$, $\eta\}$, where $\xi$ is based on the FT of elevation (as previously considered in isolation), and $\eta$ is based on both the FT of elevation and the FT of bed-slope profile. In this way, we take account of both vertical and horizontal irregularities in subglacial surfaces. We demonstrate the statistical veracity of using $\{\xi, \eta\}$ to consider roughness in terms of obstacle amplitudes and spacing, and consider the use of $\{\xi, \eta\}$ in studies of ice dynamics and subglacial geomorphological interpretation. We show that $\{\xi, \eta\}$ can be linked to basal sliding rates on the metre scale, and can be used to differentiate further than single-parameter roughness indices between different classes of subglacial landscape, in particular between erosional and depositional settings.
\end{abstract}

\section{INTRODUCTION}

Statistically characterizing the topographic structure of the basal boundary beneath ice masses is increasingly being recognized as an important imperative in glaciological research (e.g. Hubbard and Hubbard, 1998; Taylor and others, 2004; Rippin and others, 2006). Subglacial topography at all scales imparts important clues concerning the distribution of subglacial sediments, subglacial geomorphic processes and ice-stream stability (Hubbard and others, 2000; Bingham and Siegert, 2009), and is thought to exert a fundamental control on ice dynamics (Weertman, 1957; Siegert and others, 2004, 2005). Nevertheless, to date, few studies have examined subglacial topographic patterns and their influences quantitatively, perhaps due to the paucity, until recently, of appropriate bed profile datasets from contemporary glacierized environments. Modern techniques have alleviated this issue: radio-echo sounding (RES) has enabled the acquisition of myriads of bed profiles at the regional scale (decimetres to kilometres) across ice sheets and ice caps (e.g. Lythe and others 2001; Gogineni and others, 2007; Sun and others, 2009), Hubbard and others (2000) have demonstrated the ease with which centimetreand metre-scale bed profiles may be obtained across recently deglaciated terrain using microroughness meters and electro-optical distance meters respectively, and terrestrial laser scanning systems have shown great potential in surface characterization, especially with respect to threedimensional (3-D) surface characterization (e.g. Bauer and others, 2003). As 'real' bed data have thus become easier to obtain, and there is an increasing drive to integrate such data into numerical models of ice dynamics and ice-stream behaviour (Solomon and others, 2007; Pattyn and others, 2008; Hindmarsh, 2009), the need for characterizing subglacial topography in a statistically meaningful manner has arisen. In this context, recent studies, and the work described in this paper, have made use of the concept of subglacial 'roughness'.

Generally, roughness is regarded as the irregularity of a surface. In glaciology, the initial attempt at expressing subglacial bed roughness was due to the requirement of estimating how a glacier's basal sliding is controlled by the undulation of bedrock. Weertman (1957) considered an idealized case of an undulating bed as cubic obstacles with length, $\mathbf{a}$, distributed periodically with a separation distance, $\lambda$, between each obstacle. Put another way, the two parameters, $\mathbf{a}$ and $\lambda$, in this model denote the amplitude and spatial frequency of the undulations of the idealized bed. Expanding Weertman's theory to consider bedrock surfaces with randomly distributed undulations, Kamb (1970) and Nye (1970) introduced Fourier transformations (FTs) of bed elevation profiles into their theories. A FT can be used to transform any surface into a sum of several periodically corrugated surfaces, and can thus be used to express the amplitude and spatial frequency of the range of undulations present. Hence, these and follow-up studies have quantified roughness in terms of two key parameters: the vertical irregularity (amplitude) and the horizontal irregularity (spatial frequency); or in an equivalent way, as slope is the bridge between vertical and horizontal, using amplitude and slope (Paterson, 1994).

Though characterizing roughness by FT works well in theoretical dynamic studies as described above, it is not convenient for showing the spatial distribution of roughness. A basic tenet of cartography is that showing the spatial distribution of any property ideally requires that it is displayed in terms of spatial variations in the magnitude of a single parameter. Unfortunately, a full Fourier spectrum for roughness is too complicated to meet this requirement. Hubbard and others (2000) and Taylor and others (2004) circumvented this problem by defining a single-parameter 


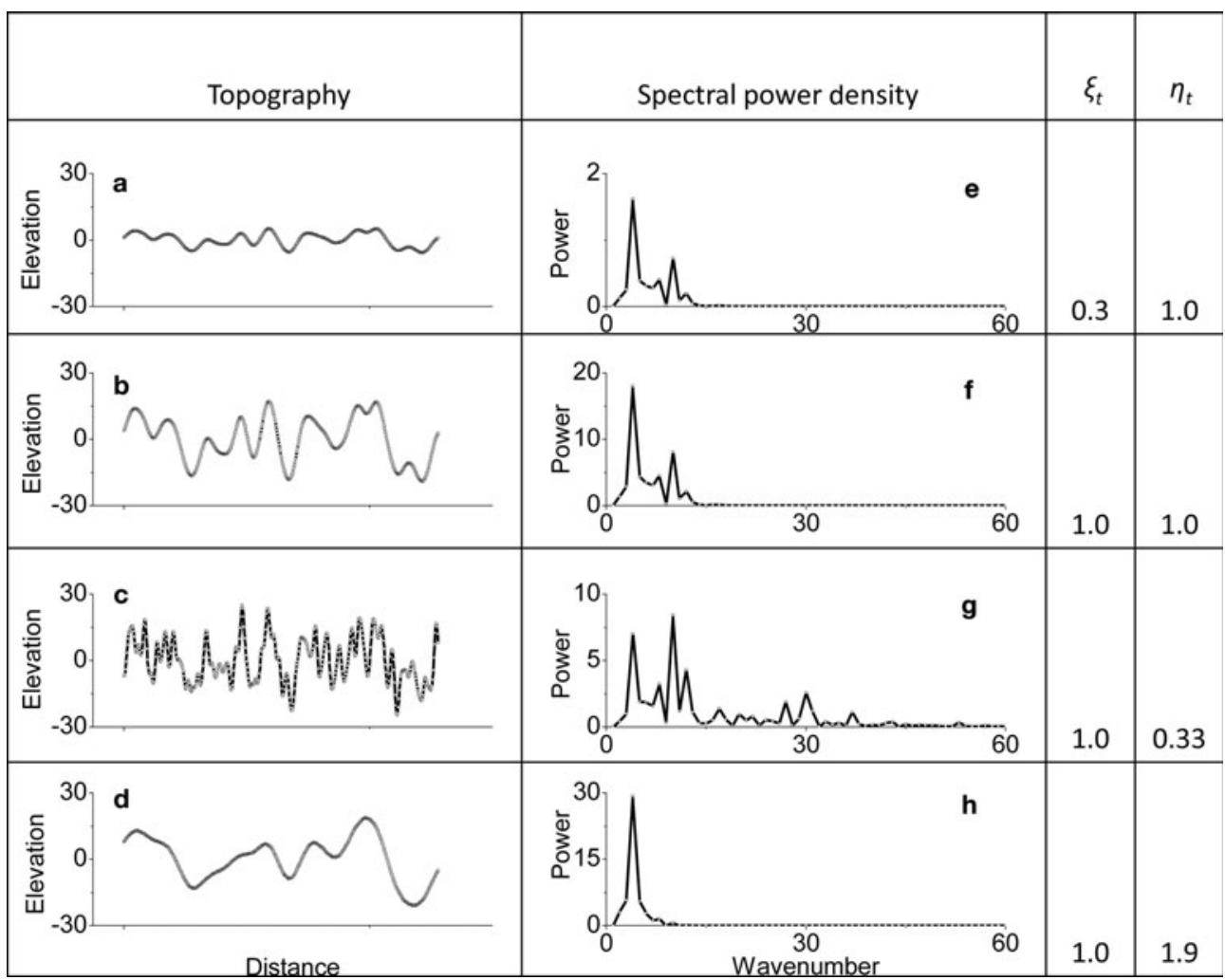

Fig. 1. Random surfaces of different roughness, generated using a self-correlation function (Thomas, 1999). (a-d) show the raw elevation profiles, and (e-h) their respective spectral power densities. All $\{\xi, \eta\}$ are in units of $\left\{\xi_{\mathrm{b}}, \eta_{\mathrm{b}}\right\}$.

roughness index defined as the integral of the spectrum within a specified wavelength interval; this method has since been applied to show the spatial distribution of subglacial roughness across Antarctica (Siegert and others, 2004, 2005; Bingham and Siegert, 2007, 2009). In this paper, we build on this method by additionally considering the FT of bedslope profiles as well as that (traditionally considered) of bedrock amplitude, and link it to horizontal irregularities. We believe that calculating the FT of both bed slope and amplitude enhances the robustness of spectral roughness techniques in subglacial landscape characterization and process inference.

\section{METHOD}

We begin by calculating the single-parameter roughness index of a profile of length, $(-1 / 2, I / 2)$. We first remove the mean, $\langle Z(x)\rangle$ from the bed elevation profile, $Z(x)$ :

$$
Z_{0}(x)=Z(x)-\langle Z(x)\rangle,
$$

where $x$ corresponds to the horizontal location and $Z$ corresponds to the raw elevation data. We then apply a FT to obtain the spectral power density, $S(k)$ :

$$
S(k)=\frac{1}{l}\left|\bar{Z}_{0}(k)\right|^{2}
$$

where $\bar{Z}_{0}(k)$ is the FT of $Z_{0}(x)$. The roughness index, $\xi$, is obtained by integrating $S(k)$ in a specified wavelength interval, $\left(k_{1}, k_{2}\right)$ :

$$
\xi=\int_{k^{1}}^{k^{2}} S(k) \mathrm{d} k
$$

When $k_{1}$ is zero and $k_{2}$ is infinity, we obtain a 'total roughness index', $\xi_{\mathrm{t}}$. This is the property used to characterize subglacial roughness across Antarctica in several previous studies (e.g. Siegert and others, 2004; Bingham and Siegert, 2009). Figure 1 illustrates this method for four rough surfaces. The surface in Figure 1a is clearly less rough than that in Figure $1 \mathrm{~b}$, and this is reflected in their $\xi_{\mathrm{t}}$ values $(0.3$ and 1.0 respectively). However, while the surfaces shown in Figure $1 \mathrm{~b}-\mathrm{d}$ all yield the same $\xi_{\mathrm{t}}$ values, they are qualitatively quite different from one another: the surface in Figure $1 \mathrm{~b}$ is steeper than that in Figure $1 \mathrm{~d}$, and that in Figure $1 \mathrm{c}$ is the steepest. We suggest, therefore, that while the parameter $\xi$ has demonstrably been useful for describing roughness, in isolation it may not be sufficient. Here we investigate the utility of considering the extra factor, the FT of bed-slope profile.

Returning to our conception of roughness in terms of vertical irregularities (amplitude) and horizontal irregularities (spatial frequency), we can interpret Figure $1 b-d$ as follows: the amplitudes of the undulations are similar, but the frequencies of the undulations differ. Hence, a factor related to horizontal irregularity needs to be introduced. Given that a bed-slope profile expresses the connection between the vertical and horizontal dimensions, it is reasonable to choose some statistical function of slope as the extra factor. A slope profile, $\mathrm{sl}(x)$, can be calculated from the elevation profile, $Z_{0}(x)$, by $\operatorname{sl}(x)=\partial Z_{0}(x) / \partial x$, and its spectral power density, $S_{\mathrm{sl}}(k)$, is

$$
S_{\mathrm{sl}}(k)=\frac{1}{l}|\overline{\mathrm{sl}}(k)|^{2}
$$

Following Equation (4), we obtain the slope index, $\xi_{\mathrm{sl}}$, by

$$
\xi_{\mathrm{sl}}=\int_{k_{1}}^{k_{2}} S_{\mathrm{sl}}(k) \mathrm{d} k
$$

Finally, as slope is the ratio of vertical dimension to 
horizontal dimension, we define the parameter $\eta$, corresponding to horizontal irregularity alone and invariant of vertical stretching of the profile, as

$$
\begin{aligned}
\eta & =\frac{\xi}{\xi_{\mathrm{sl}}}=\frac{\int_{k_{1}}^{k_{2}} S(k) \mathrm{d} k}{\int_{k_{1}}^{k_{2}} S_{\mathrm{sl}}(k) \mathrm{d} k} \\
= & \frac{\int_{k_{1}}^{k_{2}}\left(\frac{1}{T} \int_{-\infty}^{\infty} Z_{0}(x) \mathrm{e}^{-i k x} \mathrm{~d} x \int_{-\infty}^{\infty} Z_{0}\left(x^{\prime}\right) \mathrm{e}^{i k x^{\prime}} \mathrm{d} x^{\prime}\right) \mathrm{d} k}{\int_{k_{1}}^{k_{2}}\left(\frac{1}{T} \int_{-\infty}^{\infty} \frac{\mathrm{d} Z_{0}(x)}{\mathrm{d} x} \mathrm{e}^{-i k x} \mathrm{~d} x \int_{-\infty}^{\infty} \frac{\mathrm{d} Z_{0}\left(x^{\prime}\right)}{\mathrm{d} x^{\prime}} \mathrm{e}^{i k x^{\prime}} \mathrm{d} x^{\prime}\right) \mathrm{d} k} .
\end{aligned}
$$

Our two-parameter roughness index, taking into account both the vertical and the horizontal irregularities, is thus defined as $\{\xi, \eta\}$. Thus, while $\xi$ describes the magnitude of vertical deviations in the bed slope, $\eta$ quantifies the horizontal frequency of these deviations. This distinction is expressed graphically in Figure 1 (and is illuminated mathematically in section 3 ). Here each surface in Figure $1 \mathrm{~b}-\mathrm{d}$, which give the same value of $\xi_{\mathrm{t}}$, yields different values of $\eta_{\mathrm{t}}$. Figure $1 \mathrm{a}$ and $\mathrm{b}$, which have the same $\eta_{\mathrm{t}}$, have different $\xi_{\mathrm{t}}$. Figure 1 therefore demonstrates the utility of $\left\{\xi_{\mathrm{t}}\right.$ $\left.\eta_{\mathrm{t}}\right\}$ for distinguishing between all four surfaces.

As a further test, we apply our new roughness index, $\{\xi$, $\eta\}$, to a bed elevation profile acquired from East Antarctica by surface RES obtained as part of the Chinese Antarctic National Research Expedition (CHINARE) program, in 2004 (Sun and others, 2009). The profile is $\sim 200 \mathrm{~km}$ long with a spatial resolution of $70 \mathrm{~m}$ (Fig. 2). For these discrete data, summing is used in place of integration and a discrete Fourier transform (DFT) is used; a fast Fourier transform (FFT) can further be used in place of the DFT to increase efficiency. In order to consider roughness as a localized property, and to maintain consistency with earlier Antarctic roughness studies (Bingham and Siegert, 2009), we calculate $\{\xi, \eta\}$ in a moving window of length 1024 points (about $70 \mathrm{~km}$ ). The results of components $\xi$ and $\eta$ are shown in Figure $2 \mathrm{~b}$ and $\mathrm{c}$ respectively. It is apparent from Figure 2 that the two parameters describe the surface more comprehensively than each in isolation. For example, the section centred at $35 \mathrm{~km}$ has small $\xi$ and small $\eta$, reflecting lowamplitude, high-frequency roughness; whereas the section centred at $120 \mathrm{~km}$ has larger $\xi$ and $\eta$, a result of higher amplitudes but a smoother horizontal surface.

\section{STATISTICAL GEOMETRY}

To better understand the statistical meanings of $\xi$ and $\eta$ in a simple way, we consider the total roughness case, $\xi_{\mathrm{t}}$, where $\left(k_{1}, k_{2}\right)=(0, \infty)$. According to Parseval's theorem (Rudin, 1987), the value of $\xi$ can be deduced:

$$
\xi=\int_{0}^{\infty} S(k) \mathrm{d} k=\int_{0}^{\infty} \frac{1}{l}\left|\bar{Z}_{0}(k)\right|^{2} \mathrm{~d} k=\frac{1}{2 l} \int_{-\infty}^{\infty} Z_{0}^{2}(x) \mathrm{d} x .
$$

Hence the $\xi$ component of $\xi_{\mathrm{t}}$ is equal to half the mean square of the elevation profile. We calculate the distribution of the mean square of the elevation profile (Fig. 2a), and compare it with the spatial distribution of $\xi$ shown in Figure 2b. As anticipated, both distributions are the same. Similarly, $\xi_{\mathrm{sl}}$ equals half the mean square of the slope profile. This explains why $\xi$ reflects only the vertical irregularity, even though $\xi$ originates from the spectral power density which contains both vertical and horizontal information. While $\xi_{\text {sl }}$ denotes how quickly $Z_{0}(x)$ changes, component $\eta$, the ratio of $\xi$ to $\xi_{\mathrm{sl}}$, actually measures how frequently $Z(x)$ varies about its mean. This can be illustrated mathematically

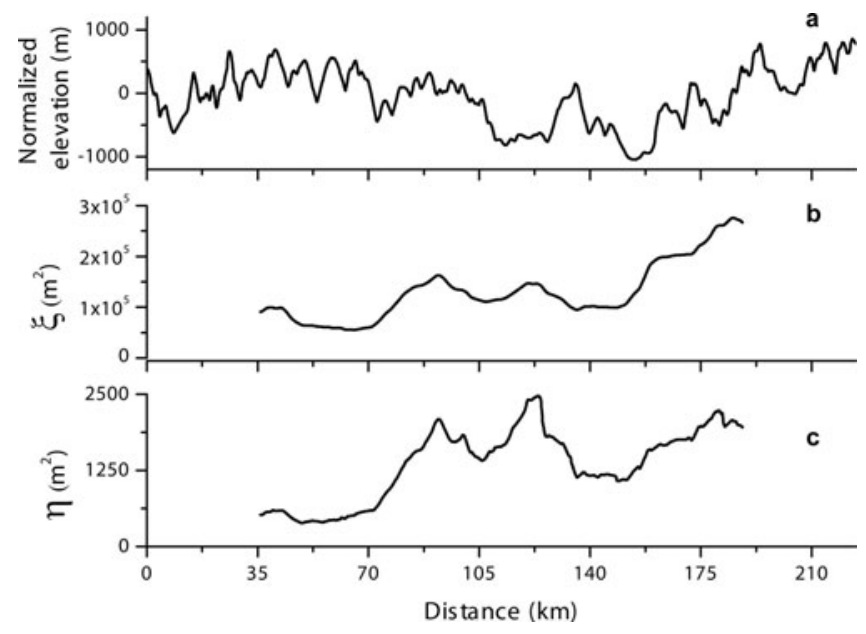

Fig. 2. (a) $\sim 220 \mathrm{~km}$ subglacial elevation profile from East Antarctica, acquired by 21st CHINARE (Sun and others, 2009). (b, c) Corresponding distribution of $\xi$ and $\eta$ respectively.

by applying Parseval's theorem to the definition of $\eta$. Consider the total roughness case,

$$
\begin{aligned}
\eta & =\frac{\xi}{\xi_{\mathrm{sl}}} \\
& =\frac{\int_{0_{1}}^{\infty} S(k) \mathrm{d} k}{\int_{0}^{\infty} S_{\mathrm{sl}}(k) \mathrm{d} k}=\frac{\int_{-\infty}^{\infty} S(k) \mathrm{d} k}{\int_{-\infty}^{\infty}\left(\frac{\mathrm{d} Z_{0}(x)}{\mathrm{d} x}\right)^{2} \mathrm{~d} x} \quad \text { (Parseval's theorem) } \\
& =\frac{\int_{-\infty}^{\infty} S(k) \mathrm{d} k}{\int_{-\infty}^{\infty} \frac{\mathrm{d} \int_{-\infty}^{\infty}(1 / 2 \pi) \bar{Z}_{0}(k) \mathrm{e}^{i k x} \mathrm{~d} k}{\mathrm{~d} x} \cdot \frac{\mathrm{d} \int_{-\infty}^{\infty}(1 / 2 \pi) \bar{Z}_{0}^{\times}(k) \mathrm{e}^{-i k x} \mathrm{~d} k}{\mathrm{~d} x} \mathrm{~d} x} \quad \text { (inverse FT) } \\
& =\frac{\int_{-\infty}^{\infty} S(k) \mathrm{d} k}{\int_{-\infty}^{\infty} \int_{-\infty}^{\infty}\left(\frac{k}{2 \pi} \bar{Z}_{0}(k)\right) \mathrm{e}^{i k x} \mathrm{~d} k \int_{-\infty}^{\infty}\left(\frac{k}{2 \pi} \bar{Z}_{0}^{\times}(k)\right) \mathrm{e}^{-i k x} \mathrm{~d} k \mathrm{~d} x} \\
& =\frac{\int_{-\infty}^{\infty} S(k) \mathrm{d} k}{\int_{-\infty}^{\infty}\left(\frac{k}{2 \pi} \bar{Z}_{0}(k)\right)\left(\frac{k}{2 \pi} \bar{Z}_{0}^{\times}(k)\right) \mathrm{d} k} \quad \text { (Parserential's theorem) } \\
& =\frac{\int_{0_{1}}^{\infty} S(k) \mathrm{d} k}{\int_{0_{1}}^{\infty}\left(\frac{k}{2 \pi}\right)^{2} S(k) \mathrm{d} k} \cdot
\end{aligned}
$$

So $\eta$ is actually a weighted harmonic average value of $(2 \pi /$ $k)^{2}$ (square of the wavelength $\lambda$ ), and the weight is $S(k)$. Thus a bed elevation profile with roughness $\{\xi, \eta\}$ means it has the same roughness index as a sinusoidal surface $Z_{0}(x)=\sqrt{2 \xi} \sin (2 \pi x / \sqrt{\eta})$. Though the horizontal irregularity can partly be shown by $\xi$ in the case of the interval of integration being divided manually into short/medium/long wavelength parts, $\eta$ is more convenient and suitable for expressing the horizontal irregularity continuously. Where two surfaces have the same total roughness component, $\xi_{\mathrm{t}}$ the surface with low $\xi$ at high frequencies tends to have larger $\eta$, while the surface with low $\xi$ at low frequencies tends to have smaller $\eta$. This is shown in Figure $1 \mathrm{c}, \mathrm{d}, \mathrm{g}$ and h. Figure $1 \mathrm{~h}$ shows that the spectral power density lies mainly in the low-frequency range, so the $\eta$ of Figure $1 \mathrm{~d}$ is larger than that of Figure 1c.

At this stage, we note some points concerning the calculation of $\{\xi, \eta\}$. Firstly, although in the case of total roughness, $\left\{\xi_{\mathrm{t}}, \eta_{\mathrm{t}}\right\}$, it would be more rapid and straightforward simply to derive the mean square instead of 
following the method outlined in section 2, we prefer our method in all other cases as it helps to discern specific wavelengths of interest. Secondly, in cases where uncertainties in the original slope profile are high, an alternative may be to use a moving mean across a small window rather than using the raw elevation profile. Thirdly, the demonstration that $\xi_{\mathrm{t}}$ is equal to the mean square of the elevation profile is valid when the elevation profile is a continuous function, but in practice this calculation will be based on a discrete set of points, and in this case the relation holds only when these points are equally spaced. Fourthly, it may well be worth noting here that this and previous analyses are all in two dimensions, largely reflecting available data as transects, but that ultimately techniques should be developed for 3-D analysis as empirical data collection improves in this direction.

\section{IMPLICATIONS FOR GLACIOLOGY}

\subsection{Ice dynamics}

On the scale of metres, bed roughness exerts a major control on an ice mass's basal motion. Weertman (1957) noted that two mechanisms operate in basal sliding: regelation and deformation. Regelation describes the process by which basal ice melts on the upstream (stoss) side of a basal obstacle and then refreezes on its downstream (lee) aspect. The difference in melting point between the up- and downstream sides results from differences in pressure exerted on each side of the obstacle by the overflowing ice, while the size of the obstacle controls the heat flux between the melting and refreezing ice on stoss and lee. Deformation is the increase in the rate of plastic flow around large bumps due to the additional stresses exerted on the adjacent ice.

The regelation-induced basal sliding velocity, $u_{1}$, deformation-induced basal sliding velocity, $u_{2}$, and total basal sliding velocity, $u$, are (Weertman, 1957)

$$
\begin{aligned}
& u_{1}=\frac{C_{1}}{a(a / \lambda)^{2}} \\
& u_{2}=\frac{C_{2} a}{\left(a / \lambda^{2}\right)^{n}} \\
& u=\frac{C}{(a / \lambda)^{n+1}},
\end{aligned}
$$

where $a$ and $\lambda$ are the average height and length of basal obstacles, $C_{1}, C_{2}$ and $C$ are factors related to non-geometric variables such as thermal conductivity and shear stress, and $n$ is Glen's flow-law exponent (Glen, 1958) whose value is discussed to be $\sim 3$ (e.g. Weertman, 1973; Russell-Head and Budd, 1979).

Based on the statistical meanings of $\xi$ and $\eta$ discussed in section 3, we can use our basal roughness index as a proxy for the vertical and horizontal length scales that are being used to estimate sliding velocity. Firstly, the interval of integration for $\{\xi, \eta\}$ should be in the metre-scale waveband. Then, given that $\xi$ is proportional to the mean square of amplitudes, a can be written as:

$$
a=\text { constant } \cdot \xi^{\frac{1}{2}} \text {. }
$$

Similarly, $\lambda$ can be written as

$$
\lambda=\text { constant } \cdot \frac{\xi^{\frac{1}{2}}}{\xi_{\mathrm{s} \mid}^{\frac{1}{2}}}=\text { constant } \cdot \eta^{\frac{1}{2}} .
$$

Hence $u_{1}, u_{2}$ and $u$ can be estimated by

$$
\begin{gathered}
u_{1}=c_{1} \xi^{-\frac{3}{2}} \eta \\
u_{2}=c_{2} \xi^{-\frac{1}{2}-n} \eta^{n} \\
u=c(\eta / \xi)^{\frac{n+1}{2}} .
\end{gathered}
$$

This method provides a way of summarily and rapidly evaluating the magnitude and distribution of the aspect of basal sliding directly attributable to basal roughness using simple statistical parameters of a subglacial bed rather than detailed topographic profiles. Other factors such as temperature (hence rate factor), possible presence of basal melt to lubricate the base, lateral stress transmitted from other parts of the ice sheet, and gravitational driving stress relating to surface and basal slopes are also important and contained in $c$. Because the scattering of electromagnetic waves relates to bed roughness (Tsang and others, 2000), obtaining simple statistical parameters of a subglacial bed requires fewer RES surveys than for detailed topographic profiles, so for subglacial beds with no detailed topographic profiles and requiring RES surveys, using our method means much fewer RES surveys are needed to evaluate basal sliding. For the areas where detailed subglacial bed profiles have already been obtained, our method requires much less computational time, and input data are needed to generate useful information relevant to basal conditions and motion processes, considered below.

\subsection{Geomorphic implications}

At the regional scale, Bingham and Siegert (2009) proposed that subglacial roughness can be interpreted at least partially in terms of the geomorphic processes operating in subAntarctic and Quaternary glaciated landscapes. Rougher subglacial topography, characterized by larger $\xi$, was tentatively associated with conditions such as continental settings, mountainous preglacial topography, cold and slow ice flow, and low rates of erosion and deposition. Smoother subglacial topography, described by lower $\xi$, was associated with marine settings, relatively flat preglacial topography, warm and fast ice flow, and high rates of erosion and deposition. However, many of these factors are interlinked and cannot be distinguished solely by $\xi$. For example, a continental setting intensively eroded by fast ice flow may have a similar value of $\xi$ to a marine setting with high deposition.

Now, using our two-parameter index, $\{\xi, \eta\}$, it may be possible to characterize and discriminate between subglacial process domains (e.g. erosional as opposed to depositional and bedrock as opposed to marine sediments) more precisely. Figure 3 shows four end-member permutations of the two indices, and their corresponding geomorphic implications are discussed below.

\section{Low $\xi$, high $\eta$}

Over a sufficient length of time, the net effect of subglacial erosion may be assumed to lower peaks and fill valleys, thereby lowering the amplitudes of bed undulations and reducing roughness but leaving the spacing between obstacles largely unchanged. Thus in erosional regions we would expect gradual lowering of $\xi$ but little change in $\eta$. By contrast, large depositional events, most commonly resulting from marine incursions, could, in theory, entirely smother the preglacial landscape, thereby affecting both $\xi$ and $\eta$. Making a direct comparison of $\{\xi, \eta\}$ between an original landscape 


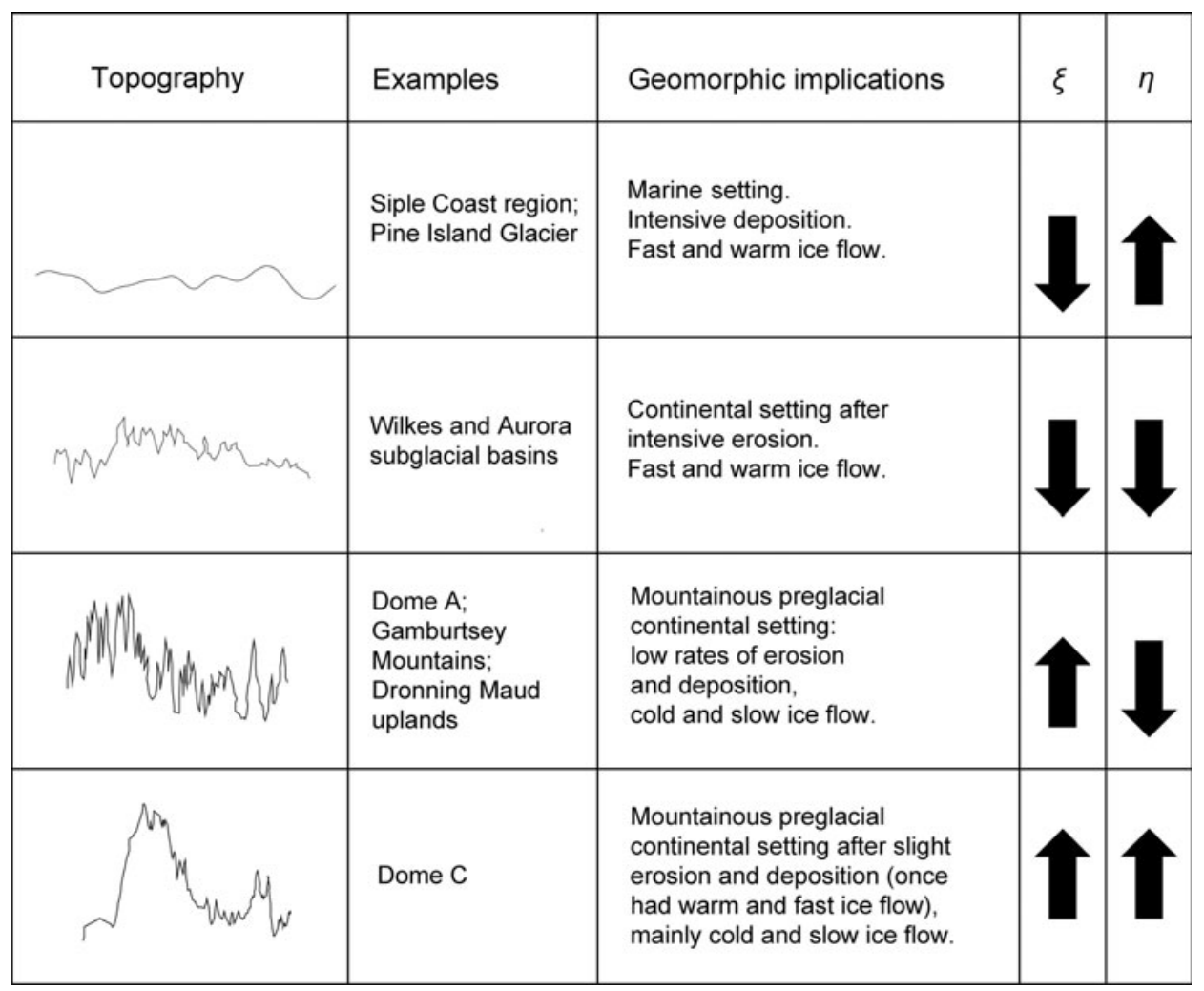

Fig. 3. Schematic framework for geomorphic interpretation of subglacial landscapes based on $\{\xi, \eta\}$. The subglacial profiles are drawn from Siegert and others (2005).

and its present 'deposited' surface is difficult, because few subglacial landscapes have been imaged below the ice/bed boundary. However, we can understand the difference between an original landscape and its present 'deposited' surface by comparing bedrock profiles with overlying englacial internal reflection horizons in RES data. Assuming an area of slow flow, thereby discounting disruption to internal layering caused by ice streaming, we can view the palaeo-landscape (bedrock) and its depositing surface (internal layers just above the bedrock) simultaneously. It is both observed and proven (Hindmarsh and others, 2006) that the depositing surface tends to parallel the long-wavelength undulations of the underlying bedrock while being insensitive to short-wavelength undulations. In such a situation, deposition demonstrably enlarges $\eta$. Therefore, we can deduce that for small- $\xi$ landscapes, those with large $\eta$ are likely dominated by deposition reflecting marine landscapes. This deduction is consistent with previous results (Siegert and others, 2005): where the bed is below sea level, supporting intensive marine preglacial deposition and postglacial reworking, $\xi$ is small, especially at high frequencies (implying $\eta$ becomes large). This subdued topography is often found beneath marine portions of both the East and West Antarctic ice sheets as a consequence of preglacial marine deposition of sedimentary material (Siegert and others, 2005).

\section{Low $\xi$, low $\eta$}

As an enlarged $\eta$ is linked to deposition, a subglacial topographic surface with small $\eta$ implies no significant deposition has occurred, so a low $\xi$ associated with this should be caused by erosion. Such a landscape therefore likely represents a continental setting influenced by intensive erosion. This deduction is consistent with previous results: where subglacial sliding is predicted by models (e.g.
Wilkes and Aurora subglacial basins, Antarctica) (Siegert and others, 2005), $\xi$ is found to be small at all frequencies (implying $\eta$ is evidently unaffected).

\section{High $\xi$, low $\eta$}

Bed roughness with high $\xi$ and low $\eta$ (i.e. high amplitude, low wavelengths) is characteristic of beds which are alpine in nature yet cold-based so that no subglacial sliding occurs. In Antarctica, such conditions have been observed beneath the ice sheet at Dome A and across the subglacial Gamburtsev Mountain Province, the subglacial landscape recording mountain glaciation during rapid ice build-up $\sim 34 \mathrm{Ma}$ ago, and the subsequent preservation of this landscape as a consequence of cold-based, low-erosion conditions predominating beneath the stable ice dome that has since overlain this region (Sun and others 2009). Subglacial uplands in Dronning Maud Land and parts of West Antarctica have similar features, their alpine-style topography having remained relatively unmodified as the ice sheet grew and the bed over upland areas became coldbased (Jamieson and others, 2010).

\section{High $\xi$, high $\eta$}

A bed retaining a high $\xi$ value suggests it has suffered neither heavy erosion nor deposition, but an enlarged $\eta$ implies there may have been some periods during which the subglacial landscape experienced deposition. Consequently, high $\xi$ with high $\eta$ could be linked to continental settings which have undergone deposition over a short period and have subsequently become protected beneath a cold and slow-flowing basal thermal regime. Such a case is presented by the Dome $\mathrm{C}$ region, where the bed was previously described as smooth at high frequencies and rough at the medium and long wavelengths (Siegert and others, 2005). 
This previous interpretation for the bed of Dome $C$ is similar to our deduction.

Between all the end-member cases described above, a variety of topographies naturally exist, all of which can be characterized quantitatively by relative values of $\xi$ and $\eta$. The particular value of quantifying bed roughness in this way is that it comprises an independent quantitative means of estimating former ice-sheet dynamics, independent of numerical ice-sheet modelling reconstructions (cf. Jamieson and others, 2010), and therefore constitutes a potentially useful tool for validating model-derived reconstructions.

\section{CONCLUSIONS}

We have extended the current use of FT methods to characterize subglacial roughness by supplementing the 'traditional' component, $\xi$ (which effectively characterizes vertical roughness), with a new parameter, $\eta$, which effectively characterizes horizontal roughness. Both parameters have dimensions of square of length. The method can be applied freely at any scale. Our analysis has shown that while (the traditionally used) $\xi$ alone forms a useful component for characterizing roughness across subglacial landscapes, the addition of the horizontal component, $\eta$, significantly enhances our ability to characterize and distinguish between different types of subglacial environment.

At the metre scale, calculation of $\{\xi, \eta\}$ represents an efficient means of characterizing the basal boundary condition for investigations of glacier dynamics, and can be used to guide interpretations of the magnitude and distribution of basal sliding. At the regional-scale, $\{\xi, \eta\}$ offers a technique for further distinguishing the factors required for gemorphological interpretation of the subglacial bed, and especially for distinguishing between subglacial landscapes of erosion and deposition.

\section{ACKNOWLEDGEMENTS}

This work was supported by the National Science Foundation of China (NSFC grants 40906100 and 40476005), National High Technology Research and Development Program of China (2008AA121702), Polar Science Strategy Research Foundation of China (grant 20080204), China Postdoctoral Science Foundation (CPSF grant 20090450085), National Basic Research Program of China (973 Program 2010CB950301) and UK Natural Environment Research Council grant NE/D003733/1.

\section{REFERENCES}

Bauer, A., G. Paar and V. Kaufmann. 2003. Terrestrial laser scanning for rock glacier monitoring. In Phillips, M., S.M. Springman and L.U. Arenson, eds. Permafrost. Lisse, Swets \& Zeitlinger, 55-60.

Bingham, R.G. and M.J. Siegert. 2007. Radar-derived bed roughness characterization of Institute and Möller ice streams, West Antarctica, and comparison with Siple Coast ice streams. Geophys. Res. Lett., 34(21), L21504. (10.1029/2007GL031483.)

Bingham, R.G. and M.J. Siegert. 2009. Quantifying subglacial bed roughness in Antarctica: implications for ice-sheet dynamics and history. Quat. Sci. Rev., 28(3-4), 223-236.

Glen, J.W. 1958. The flow law of ice: a discussion of the assumptions made in glacier theory, their experimental foundation and consequences. IASH Publ. 47 (Symposium at Chamonix 1958 Physics of the Movement of the Ice), 171-183.
Gogineni, S. and 11 others. 2007. Polar radar for ice sheet measurements (PRISM). Remote Sens. Environ., 111(2-3), 204-211.

Hindmarsh, R.C.A. 2009. Consistent generation of ice-streams via thermo-viscous instabilities modulated by membrane stresses. Geophys. Res. Lett., 36(6), L06502. (10.1029/2008GL036877.)

Hindmarsh, R.C.A., G.J.M. Leysinger Vieli, M.J. Raymond and G.H. Gudmundsson. 2006. Draping or overriding: the effect of horizontal stress gradients on internal layer architecture in ice sheets. J. Geophys. Res., 111(F2), F02018. (10.1029/ 2005JF000309.)

Hubbard, B. and A. Hubbard. 1998. Bedrock surface roughness and the distribution of subglacially precipitated carbonate deposits: implications for formation at Glacier de Tsanfleuron, Switzerland. Earth Surf. Process. Landf., 23(3), 261-270.

Hubbard, B., M.J. Siegert and D. McCarroll. 2000. Spectral roughness of glaciated bedrock geomorphic surfaces: implications for glacier sliding. J. Geophys. Res., 105(B9), 21,295-21,303.

Jamieson, S.S.R., D.E. Sugden and N.R.J. Hulton. 2010. The evolution of the subglacial landscape of Antarctica. Earth Planet. Sci. Lett., 293(1-2), 1-27.

Kamb, B. 1970. Sliding motion of glaciers: theory and observation. Rev. Geophys. Space Phys., 8(4), 673-728.

Lythe, M.B., D.G. Vaughan and BEDMAP consortium. 2001. BEDMAP: a new ice thickness and subglacial topographic model of Antarctica. J. Geophys. Res., 106(B6), 11,335-11,351.

Nye, J.F. 1970. Glacier sliding without cavitation in a linear viscous approximation. Proc. R. Soc. London, Ser. A, 315(1522), 381-403.

Paterson, W.S.B. 1994. The physics of glaciers. Third edition. Oxford, etc., Elsevier.

Pattyn, F. and 20 others. 2008. Benchmark experiments for higherorder and full-Stokes ice sheet models (ISMIP-HOM). Cryosphere, 2(1), 95-108.

Rippin, D.M., J.L. Bamber, M.J. Siegert, D.G. Vaughan and H.F.J. Corr. 2006. Basal conditions beneath enhanced-flow tributaries of Slessor Glacier, East Antarctica. J. Glaciol., 52(179), 481-490.

Rudin, W. 1987. Real and complex analysis. Third edition. Singapore, McGraw Hill.

Russell-Head, D.S. and W.F. Budd. 1979. Ice-sheet flow properties derived from bore-hole shear measurements combined with icecore studies. J. Glaciol., 24(90), 117-130.

Siegert, M.J., J. Taylor, A.J. Payne and B. Hubbard. 2004. Macroscale bed roughness of the Siple Coast ice streams in West Antarctica. Earth Surf. Process. Landf., 29(13), 1591-1596.

Siegert, M.J., J. Taylor and A.J. Payne. 2005. Spectral roughness of subglacial topography and implications for former ice-sheet dynamics in East Antarctica. Global Planet. Change, 45(1-3), 249-263.

Solomon, S. and 7 others, eds. 2007. Climate change 2007: the physical science basis. Contribution of Working Group I to the Fourth Assessment Report of the Intergovernmental Panel on Climate Change. Cambridge, etc., Cambridge University Press.

Sun, B. and 8 others. 2009. Gamburtsev mountains and the origin and early evolution of the Antarctic Ice Sheet. Nature, 459(7247), 690-693.

Taylor, J., M.J. Siegert, A.J. Payne and B. Hubbard. 2004. Regionalscale roughness beneath ice masses: measurement and analysis. Comput. Geosci., 30(8), 797-908.

Thomas, T.R. 1999. Rough surfaces. Second edition. London, Imperial College Press.

Tsang, L., J.A. Kong and K.-H. Ding. 2000. Scattering of electromagnetic waves: theories and applications. New York, Wiley.

Weertman, J. 1957. On the sliding of glaciers. J. Glaciol., 3(21), 33-38.

Weertman, J. 1973. Creep of ice. In Whalley, E., S.J. Jones and L.W. Gold, eds. Physics and chemistry of ice. Ottawa, Ont., Royal Society of Canada, 320-337. 